\title{
L'ATTORE E LA STRADA
}

Paolo PUPPA ${ }^{1}$

- RIASSUNTO: Excursus sull'teatro politicizzato dal '68 alla fine degli anni '80. Animazione nelle scuole e nelle fabbriche. Inventario delle tournées americane in Italia. Scoperta del terzo teatro. Dario Fo e lo spostarsi dello spazio. Fine della militanza politica sostituita dall'imperialismo mediatico. L'attore narratore nella svolta deideologizzante.

- PAROLE-CHIAVE: Teatro politico; terzo teatro; Dario Fo.

Si possono forse inventariare, intorno al tema dell'attore e della strada, o meglio dell'attore e della piazza, tipologie diverse ma tutte riconducibili all'aura che si sprigiona dal ' 68 . Solo che man mano che invecchio comincio in qualche modo a deideologizzarmi. Così, qualche anno fa ero convinto che questo ' 68 segnasse un evento epocale, sinonimo di svolta palingenetica, un po' come era stato il ' 45 , non solo rispetto al teatro ma anche alla società tutta. Adesso, invece, ipotizzo che fra qualche decennio nella prospettiva presbite dei posteri certe sopravvalutazioni non solo ideologiche ma tecnico-artistiche, relative alla scena del ' 68 , rischieranno di essere ridimensionate. Anche il rapporto che il teatro intrattiene colla cultura in generale è mutato negli ultimi trentanni. Allora, in quell'ormai lontano tempo, la televisione durava poche ore, disponeva di un solo canale. Il libro possedeva una forza, una presenza magnetica per i giovani che non può più esibire oggi. Se si fa un'inchiesta, su quello che leggono gli studenti attualmente, infatti,

1 Facoltà di Lingue e di Letterature dell'Università di Venezia - 30124 - Venezia - Italia. 
emergono risposte sorprendenti sulla caduta del numero dei libri letti rispetto a trentanni fa. Il numero di ore che un ragazzo passa davanti ad un televisore, confrontato col numero di ore dedicato alla lettura, fornisce un quadro sinistro. Anche la capacità di tenuta dell'attenzione del ragazzo su di un testo è di molto attenuata. Insomma, nell'organizzazione multimediale il teatro ricopre solo un minimo spazio. Gli altri media interagiscono e lo rendo no precario, marginale. Certo, in altri paesi, il processo di subalternità della scena trova qualche ostacolo, e l'imperialismo televisivo appare meno irreversibile. Nei paesi anglosassoni, ad esempio, tutti fanno teatro, non importa se lo fanno male. Perché il teatro serve per sbloccare la timidezza, per vincere la balbuzie, per inventarti gli altri io che sono in te.

Eppure, quello che rimpiango di quel periodo vicino/lontano è l'investimento escatologico garantito al palcoscenico. Ora, l'etimo della parola utopia, seguendo il polacco Bronislaw Baczko, oscilla tra ou-topos ed eu-topos, cioè da un lato un luogo bello, eu, e dal l'altro un luogo che non esiste, ou. Ebbene, si può sostenere che la cultura sedimentata attorno al ' 68 ha scambiato tra loro proprio l'eu e l'ou. Innanzitutto si è all'improvviso interrogato il teatro con domande del tipo: "a chi, perché, dove, quando, faccio teatro?". Tutte domande caratterizzate da una curiosità fenomenologica sulla essenza della scena.

Le avanguardie precedenti, si consideri l'epoca tra la fine dell' $800 \mathrm{e}$ il primo ' 900 , hanno per certi aspetti trascurato questi interrogativi. Uno dei grandi maestri di questa epoca, Peter Brook, si pone il paradosso lancinante e spietato: "se il teatro scioperasse, se il teatro sparisse all'improvviso, chi se ne accorgerebbe, chi ne soffrirebbe?". Ci si accorge brutalmente della drammatica superfluità del teatro, della sua eccedenza, della sua marginalità, necessaria nell'industria dello spettacolo riproducibile e nell'era informatica. Finita, e per sempre, la centralità della scena, antico polmone, mente e cuore della polis, strumento processuale e cognitivo, divertissement dei principi e dei ceti bassi nelle epoche d'oro del teatro. No, il palcoscenico non è più necessario. Tanto vale andare in piazza, sulla strada. Ecco, il ' 68 teatrale, cerca di reagire alla scoperta traumatica della propria irrilevanza entro il sistema delle comunicazioni. Da ciò deriva, altresì, e portata avanti dal momento utopico più alto e radicale dello stesso ' 68 , la contrapposizione al detto sistema, specie alla televisione che pure non era pervasiva e totalizzante come oggi. Anzi, potremmo dire che se i mass-media sono le lingue della distanza, del padre, la scena è l'ultimo dialetto che rimane in quanto è la lingua della fisicità maternale, della vicinanza all'altro, al corpo dell'altro finalmente ritrovato. 
Il corpo inizia a liberarsi proprio grazie a Jerzy Grotowski, entro un orizzonte cristiano-slavo in cui l'archetipo cristologico, sotteso nella memoria moderna anche laica, si incrocia col cattolicesimo polacco antagonista nei confronti del dominio marxista-filosovietico. Perché nella Polonia di quegli anni essere cristiani e portare avanti un teatro mistico al tempo dello stalinismo comporta rischi personali e carismi trasgressivi. Ora Grotowski nella misura in cui porta quale tema il corpo provoca polemiche e dissensi nei suoi rapporti col coevo teatro di sinistra di quegli anni, basti pensare alle feroci stroncature subite ad opera di Roger Planchon, di Paolo Grassi e di Giorgio Strehler, tutti schierati dalla parte, considerata opposta, di Brecht. Anche perché Grotowski fa coincidere le scelte drammaturgiche coll'ambivalente complicità tra carnefice e vittima. Ecco pertanto testi, anche classici, rigiocati e manipolati su variazioni fisiche che mettono in evidenza la compresenza in tutti noi del ruolo di carnefice e di vittima. Quindi, una fortissima connotazione agiografica. I corpi dei suoi attori sono corpi che ripropongono lo scandalo del Golgota, cioè si rimettono in campo per offrirsi in un sacrificio non solo simbolico. Impliciti i richiami ad Artaud, così come artaudiana risulta la metafora del corpo che brucia in scena, col pubblico voyeur che scansa le scintille. Ma la strategia è quella di un legame intensificato colla sala, nell'asse attore/spettatore, io/tu che potremmo definire faticoconativo, prelevando categorie dalla linguistica. Negli ultimi anni della sua vita, Grotowski non ha prodotto più teatro in senso professionale. Anzi, si pensi a Marcel Deschamp nel settore dell'arte concettuale, o a Rimbaud che vendeva armi in Africa, ha chiuso colla propria arte e ha mutato mestiere. O meglio, dopo aver raggiunto col Principe Costante una specie di perfezione strutturale, non soddisfatto del successivo Apokalipsis cum figuris, ha detto basta. Ha privilegiato una strategia transazionale, sul rapporto appunto io-tu. E quindi col suo gruppo ha costruito incontri allo scopo di svegliare nell'altro la capacità di fare esperienza, di evitare la ripetizione di se stesso. Quindi, solo progetti a tempo lungo, che riguarda vano la sfera della relazione intersoggettiva gestita da professionisti un po' sacerdoti-terapeuti, un po' analisti che curano e rilanciano l'attitudine interpersonale dei partecipanti agli stages. Al tempo dei suoi spettacoli, allorché faceva ancora teatro, per lui condizione irrinunciabile era nondimeno che ci fossero pochi spettatori a contatto fisico cogli interpreti, magari costretti ad indossare i medesimi costumi di questi ultimi, come nel Faust da Marlowe. L'estrema contiguità assicurata, a ridosso del fiato e del sudore degli attori, sempre in scena quali macchine pulsanti, consentiva alla microsala di percepire la varietà estrema di segnali inviati dal corpo dei performer, divenuti di fatto un'autentica polifo- 
nia. In effetti, per Grotowski il teatro non è se non un incontro traumatico, in grado di scrostare abitudini mentali, pregiudizi, sicurezze inveterate, in grado ancora di rimettere in moto le nostre stesse virtualità creative per quanto concerne la dinamica dei nostri comportamenti quotidiani, scavando nella nostra coscienza e nella nostra percezione del reale.

Anche il pugliese Eugenio Barba, allievo di Jerzy Grotowski, si accosta alla tradizione orientale, nel suo caso in termini specifici all'indiano Katakali, sbarazzandosi della propedeutica accademica dei nostri attori, della recitazione di testa. Gli attori italiani gli appaiono limitati nell'uso della propria voce, e per di più una voce impostata dalla dizione toscanocentrica. Mentre il suo interprete, per rifarci al modello grotowskiano, deve essere in grado di manifestare anche una grammatica del piede, di emettere un suono occipitale che renda espressivo anche l'alluce. Perché adesso, con lui, tutto il corpo è suonato dall'alluce fino alla punta dei capelli. Quindi nella danese Holstebro, emula del laboratorio polacco a Wroclaw, Barba costituisce il suo falansterio, dove l'attore vive a tempo pieno apprendistato e preparazione di spettacoli. E si tratta di performer creativi, occorre ribadirlo, dimensione non certo nuova, prelevata pur sempre da alcune anticipazioni delle avanguardie da Stanislavskij a Copeau. Quale la funzione del regista? Quella di spartitraffico tra motivi creativi proporsti dagli attori, di modo che l'attore non risulti un mero impiegato, un funzionariop passivo e diligente come spesso avviene nei nostri Stabili. Nel laboratorio, al contrario, l'accento verte sul diritto - dovere dell'interprete di scriversi la propria parte, ritagliata a ridosso del corpo mentre il regista è uno che osserva e seleziona, che taglia e cuce. Quindi, attore a tempo pieno, attore creativo, attore socializzato nel falansterio, attore terapeuta contrapposto dialetticamente ad uno spettatore pigro, annoiato che deve essere svegliato. Barba porta avanti un altro aspetto, il baratto. Questo gruppo, infatti, esporta spettacoli dopo una lunga preparazione, (si pensi ai nostri famigerati quaranta giorni di prove!) e vive e produce lentamente un prodotto che nasce da continui adattamenti e rinnovamenti. A tale perfezionismo mistico poi si unisce il movimento verso aree decentrate, emarginate, aree in qualche modo non toccate dall'industria culturale. Qui sta il potlàc, nel senso antropologico di scambio simbolico: io porto a te una cosa nostra, e voi cosa mi date? Canzoni, danze, elementi folk, che vengono ravvivati e rimessi in fase grazie a questa transazione dolcemente provocatoria. Quale supporto bibliografico per questo attore esotico-cosmopolita, stanno gli studi precedenti di Ernesto De Martino, si pensi a Sud e magia del '59. Ma l'attore sacro, realizzato da simili falansteri, presenta indub- 
bie analogie con altri versanti dell'antropologia, dove l'interprete viene appaiato all'isterico, si pensi a Clefs pour l'imaginaire di Octave Mannoni del ' 57 , al posseduto da forze occulte, in grado di effettuare escursioni psichiche, al limite di incontrare i morti. Anche con Barba, l'attore deve continuamente mettere in gioco le grandi ambiguità del proprio sottosuolo inconscio. Se Grotowski insisteva sulla complicità tra carnefice e vittima, a sua volta Barba opera, sulla scia di Mejerchol'd, un montaggio emotivo di gesti, tra antipatia e simpatia, nei riguardi della dramatis persona da recitare: occorrono pertanto nello spazio gesti che evidenzino una gioiosa e tragica confusione ideologica verso ciò che viene mostrato.

Dopo il corpo liberato, un secondo corpo, quello esperanto. Ci spostiamo dalla Polonia e dalla Danimarca, da Wroclaw e da Holstebro, così come dal tarantolismo pugliese-lucano, per giungere a Londra. Prima ho citato Peter Brook. Ebbene, la Londra degli anni ' 60 ospita due grandi spettacoli drammatici che vengono ben prima del ' 68 . Uno è Marat-Sade, che Peter Brook costruisce da un testo del '64 di Peter Weiss. Qui, entro il manicomio di Charenton nel tempo del dopo Rivoluzione francese, i pazienti simulano l'uccisione di Marat per opera di Charlotte Corday, diretti dal più illustre dei degenti, il Marquis de Sade. Il tema del teatrofollia si coniuga in tal modo col play within play, in una sorta di discussion play su due atteggiamenti antitetici, il principio di piacere, rappresentato dal Marchese, e quello del reale, condensato nelle farneticazioni utopiche di Marat. In questo caso Artaud e Brecht dimostrano la reciproca compatibilità in scena. Due anni dopo, nel '66, ecco un nuovo spettacolo di Brook, apparentemente meno importante eppure molto significativo, ovvero US, nel doppio senso di noi e United States. Al centro, uno dei temi ossessivi di quegli anni, la guerra nel Vietnam, coi bombardamenti al Napalm dei bambini innocenti su cui tutta la sinistra, manifestava la propria rabbiosa impotenza. Sul palcoscenico, alla fine, si avanza un attore che tiene tra le mani una farfalla viva che, guardando fissamente il pubblico, ammazza all'improvviso. Subito reazioni furibonde da parte della sala. Ma in questo modo si mette in evidenza la grande contraddizione tra l'ideologia e la pratica quotidiana, nel senso che a teatro un gesto non simbolico, ma reale, pretende altre reazioni da quelle consuete. Ed è una povera farfalla fatta a pezzi che smaschera così lo spettatore impegnato, nelle sue laceranti mistificazioni. Ma perché ho detto corpo esperanto? Per il fatto che Brook riunisce attori appartenenti a razze, a lingue diverse e ogni volta li costringe a recitare nella lingua della committenza. Cioè se è l'Inghilterra che finanzia, sarà la lingua inglese, se è la Francia (negli ultimi anni Brook lavora alle Bouffes du Nord, sala della periferia parigina) sarà il francese, e sempre una koiné 
mal pronunciata, meteca, rispetto ai criteri puristi della bella pronunzia. Del resto, questa lingua improvvisata, superficialmente conosciuta dalle varie etnie che interagiscono, viene supportata dal corpo. Si ribadisce nella pratica il fatto che noi comunichiamo innanzitutto con il corpo. Le nostre emozioni sono prelinguistiche, extragrammaticali, per la fortissima capacità sfruttata dal fisico proprio per sopperire alle differenze linguistiche. Il progetto di Brook, altresì, è quello di andare contro la noia teatrale. Perché la gente a teatro si annoia, perché il teatro uccide nel pubblico il desiderio poi di tornarci volentieri. Per scongiurare la noia, Brook propone da un lato pièces antropologiche sull'Africa, e dall'altro il ritorno ai classici. Shakespeare che in Inghilterra è autore nazionalpopolare, cioè conosciuto radicalmente, recitato nelle scuole, dagli asili alle università, viene tutto scrostato, liberato dalla abitudini percettive e recitative, mostrato quasi fosse la prima volta. Ma Shakespeare, a sua volta, nella rilettura agiografgica di Brook, mette assieme il rutto alla preghiera, cioè metabolizza la tendenza metafisica all'invisibile, all'aldilà, colla biologia, gli escrementi. In una parola, il teatro rozzo e il teatro sacro, formula che mette assieme una convivenza quasi cubista di registri, garantendo una varietà polimetrica non solo linguistica ma prospettica. In Shakespeare, dice sempre Brook, non c'è mai una verità, ci sono tanti diversi personaggi ognuno dei quali ha una sua idea, una sua interpretazione. Potremmo sostenere che pirandellianamente Shakespeare non sceglie, ma lascia tutto aperto, non finito.

Terzo corpo, il corpo di strada. Qui mi riferisco alle grandi tournées americane in Europa. Dal 1964 sino al '68, il Living Theatre porta in giro, dalla Francia all'Italia, le sue performance stordenti, il funambolico e irriverente Mysteries and Smaller Pieces, l' Antigone sconvolgente, il Frankenstein catastrofico, il Paradise now per ultimo, che ad Avignone, durante il festival del maggio ' 68 , coinvolge e travolge anche il direttore, Jean Vilar, fermo al suo concetto rollandiano di teatro popolare e interclassista, impreparato alla radicalizzazione dello scontro tra scena e ordine pubblico. Il suo festival, in precedenza, aveva declinato un teatro all'insegna di servizio pubblico, di potage offerta alla massa, con scelte culturali che avevano fecondato in Italia i nostri Grassi e Strehler. Il Living non fa altro che riproporre in termini più violenti le antiche querelles con Jean Paul Sartre che negli anni '50 rinfacciava all'animatore l'assenza di pubblico proletario dalle sue sale. Paradise now viene preparato nell'isolamento solare di Cefalù, in un orizzonte che mescola Herbert Marcuse e Wilhelm Reich, Bakunin e l'orientalismo tantrico. Il mito della rivoluzione sessuale, la possibilità che nel teatro ci si possa liberare dalle pulsioni, è una delle varie utopie che la scena del Living intende 
realizzare sul palcoscenico, in una forte investimento didattico. Come può l'educazione far dimenticare che il nostro corpo è una fonte di piacere? Perché privarci di questo piacere? Paradise now costruisce una serie di ascensioni, di elevazioni, di passaggi iniziatici verso il motivo escapistico e lievitante, invitando il pubblico ad imitare gli attori. Jean Vilar esigeva, da regolamento aziendale, il rispetto delle regole, come ad esempio il pagamento del biglietto. Al contrario, il gruppo americano sollecitava la sala a non ubbidire e ad organizzare lo spettacolo fuori, nella strada. Impossibile comunicare tra pratiche e idee tanto antitetiche di spettacolo. D'altra parte, il mito del teatro di strada, l'utopia on the road non può certo dirsi un'invenzione del Living, in quanto recupera la grande memoria dell'Agit prop, del teatro di controinformazione che nella Germania di Weimar negli anni prima dell'avvento nazista, costruiva, mentori Benjamin ad Asja Lacis, gruppi di attori operai e dilettanti, a volte impegnati davanti al cinema in cui si rappresentavano pellicole sentimentalistiche, per farne la parodia, e per denunciarne la funzione smobilitante nei confronti delle facoltà critiche del pubblico piccolo-borghese. Ora, i temi veicolati dal Living riguardano spesso il denaro e la repressione, grazie a parole seriali, alla ripetizione di pochi suoni, come "stop the war". Nel frattempo, le tournées del Living rifunzionalizzano i testi di Richard Schechner e di Guy Debord, ossia registi e teorici sul teatro di partecipazione, sulla fine della sala all'italiana e sulla moltiplicazione dello spazio ludico-partecipativo. Di fatto, un'appendice delle novità futuriste che avevano già anticipato il palcoscenico simultaneo $\mathrm{e}$ compenetrato.

Quarto corpo, quello pedagogico o di quartiere. In questo caso si abbassa la tensione mistica, idealistica e si rientra in una dimensione minimalista: quartieri, scuole elementari, ma anche carceri, manicomi. Un gruppo teatrale, magari non professionale, fa dell'animazione o della controinformazione, seguendo non tanto il modello weimariano o sovietico, ma francese, ossia la pedagogia di Rousseau, e i miti illuministi-romantici della microfesta. Il teatro è un evento piccolo che si fa in un'area possibilmente omogenea, per liberare un determinato territorio o per dare ad esso la coscienza della sua storia, del suo passato, del suo futuro. Si consideri, ad esempio, Giuliano Scabia allorché questo teatrante escogita nel ' 72 assieme allo psichiatra Franco Basaglia l'avventura di Marco Cavallo, sorta di cavallo di Troia che contribuisce a smantellare il manicomio triestino. Momento simbolico per eccellenza, quando i pazienti coinvolti nell'esperienza appendono sul cavallo i propri feticci, oggetti, fogli, amuleti, ciò che li fa essere persone distinte, e in un secondo momento portano lo stesso cavallo in trionfo per i padiglioni liberati 
dell'Istituto. Certo, a rileggere l'avventura nel volume-diario scritto da Scabia per l'Einaudi si fatica a trattenere l'irritazione davanti all' euforia del racconto, trasformato nell'agiografia festiva del matto, un po'seguendo la pubblicistica sulla schizofrenia di Deleuze e Guattari. Meglio i più discreti e umili reportages di un altro animatore, che opera di preferenza nel mondo dell'infanzia, ossia Remo Rostagno. Maestro elementare al lavoro nel circuito periferico torinese, Rostagno allestisce con i suoi studenti degli spettacoli collages, tra cui il delizioso Sul cartello era scritto vietato l'ingresso, oppure un poetico-grottesco rifacimento dal Petit prince di Saint-Exupéry. In entrambi i casi, si registrano preliminarmente le frasi repressive dei genitori della cintura piccola borgheseproletaria e lo spettacolo funziona allora quale garbata provocazione verso simili depositi ideologicia. Gli utenti sono i famigliari stessi, invitati nella scuola ad assistere ad un work in progress, scandito per stazioni, e sono i bambini che fanno strada, che aiutano i genitori a scoprire i riflessi delle loro fobie, delle loro ossessioni nei cartelloni, nei disegni, nelle registrazioni al magnetofono, nelle parole orripilanti e repressive. Un'apparente scuola Montessori, in realtà una spietata controeducazione. All'interno di un simile circuito, si installa altresì la parte migliore, a mio parere, di Dario Fo. Al di là del mitico Mistero Buffo, autentico manifesto-spettacolo sessantottino che quasi anticipa le recenti convergenze tra cattolicesimo impegnato e sinistra riformista in Italia, sta infatti il Fo impegnato entro i circuiti Arci, con performance da porta accanto, in cui un gruppo di attori anonimi si accasa in un ambiente specifico per studiarne le contraddizioni, e poi mostrargliele in una sorta di teatro dello specchio. Le polemiche feroci, scatenate intorno a L'operaio conosce trecento parole, il Padrone mille. Per questo il Padrone è il Padrone, oppure Legami pure che tanto spacco tutto lo stesso, mostrano i ricatti ideologici esercitati dalle regioni "rosse", dissociate tra la militanza pubblica del compagno e il suo privato comportamento (a casa, tra le pareti domestiche, magari l'operaio, sfruttato dalla fabbrica, è divenuto un piccolo padrone delle ferriere, grazie al lavoro a cottimo).

Nel frattempo, sempre più sembra svalutata la prola scritta, svalutata, utilizzata quale mera appendice del corpo. La nuova drammaturgia comincia lentamente a formarsi dal " 61 , allorché cade la censura preventiva grazie alle polemiche suscitate dall'Arialda di Giovanni Testori colla regia di Luchino Visconti. Ora non sussiste più il controllo di un un commissario di polizia prima del debutto di un testo. Questo fatto ridimensiona il fortissimo verbocentrismo precedente, perché adesso la parola non è più vincolata dalla censura stessa. A poco a poco si inseriscono, anche se in ritardo, altre forme di drammaturgia, come viene sancito dal 
famoso congresso di Ivrea del '67. Qui, alcuni teorici e scrittori delle neo-avanguardie si riuniscono per favorire una écriture multidimensionale, non più verbocentrica. L'indubbia svalutazione verso la parola scritta si coniuga col recupero altresì della grande letteratura teatrale scritta di quegli anni. Dilagano in Italia le traduzioni di Pinter, di Adamov, di Beckett che mostrano in concreto come quando si parla, si mente o si celano gli aspetti essenziali del reale, che solo il corpo potrebbe tentare di rendere. Dunque, un parola inadatta o menzognera. Le neoavanguardie propongono pure la drammaturgia del pastiche, il dialetto culto, non quello nostalgico-perbenista alla Eduardo De Filippo, ma quello trasgressivo-notturno, tra perversioni e travestismi alla Enzo Moscato o alla Annibale Ruccello, a Napoli, o alla Franco Scaldati a Palermo. Questa drammaturgia finalmente bilingue e regionalista fiorisce anche nella Toscana di Ugo Chiti, nel Veneto del più recente Marco Paolini. Un altro versante di questa parola ridotta, o fisicizzata comprende commediografi minimalisti, sulla scia di Flaiano e della Ginzburg, dove, finalmente, per evitare gli eccessi della lingua di piombo, ossia iperscritta, si individuano fasce sociali determinate, aree impiegatizie, sportive, tra mansionari specifici e gergalità tecniche, e si cerca di perimetrare o ridurre il lato generalizzante della lingua stessa.

Quinto corpo, quello frigido, omologato a immagine, mentre svapora ogni tensione escatologica, e si abbandona qualsiasi contaminazione colla strada. Tanto più che il corpo immagine si allea con i mass-media, nel trionfo della visualizzazione tecnologica attraverso spettacoli virtuali-elettronici. E sono sempre le tournées europee di gruppi americani, agli inizi degli anni'70 a suggellare il successo delle nuove spinte. Tra costoro, il più carismatico, il più astuto, anche per il suo coinvolgere patologie e handicap, il più seducente è certo Bob Wilson, gran mago della schizofrenia percettiva, inventore di raffinati congegni anamorfici, tra allucinanti slow motion ed algidi cartoons. Al contrario del Living Theatre, di Joe Chaikin, di Peter Brook, il corpo non è più esperanto, non sa né intende più ridurre le distanze, ma si fissa, si vetrifica quasi ad icona. Qui, si ripescano persino Craig ed Appia, vale a dire i precursori di una scena onirica-enigmatica in cui la luce e le forme costituiscono gli autentici protagonisti del casting audiovisivo, precedenti rigorosi delle serialità sonore e iconiche del regista texano. Il sogno, adesso, monta in scena, allo stesso modo d'un altro versante, quello polacco di Tadeusz Kantor. Stavolta, la Polonia non è quella mistica di Grotowski, ma quella cinica new dada della Classe morta del 1975, sorta di post-happening cimiteriale in cui il corpo dell'attore viene doppiato dal manichino. Il legno, metonimico della croce, veicola il motivo del sacrificio ma in chi- 
ave ludica e astratta. L'antico dualismo tra organico e inorganico, proprio della Bauhaus, sembra pacificarsi in soluzioni ironicamente regres sive.

Infine, il corpo solitario. Spesso, l'attore solo sulla piazza da militanteagente ideologico che era nel periodo della contestazione e dell'utopia, diviene la star musicale, il performer rock, in grado di soggiogare folle ai suoi piedi, più e meglio dei Fo d'annata. Ecco allora l'esplosione dei grandi chansonniers, degli attori monologanti, del neocabaret televisivo, tra Gaber, Grillo e Moni Ovadia, i quali proseguono senza le tinte maledettiste, senza il côté sublime di Carmelo Bene. E quest'ultimo, a sua volta, magari recitando Dino Campana o Majakovskij, tenta col proprio virtuosismo vocale, sorretto magari da protesi elettroniche, di emulare la musica. Perché la voce, in questo caso, non é più quella centralizzata e bene impostata dalle scuole tradizionali di recitazione, ma è un suono dilatato verso un assoluto astratto, verso il nichilismo. Ma anche se lasciata, la strada però continua ideal mente ad agire in altri versanti dell'attore monologante, magari maturato attraverso l'animazione nelle scuole, a contatto coi bambini, come Marco Baliani o Marco Paolini, per restare all'Italia, capace di costruire autentici filo, ovvero racconti maliosi. E non mancano moduli brechtiani nella scansione tra dialogo col pubblico e la fabula recitata, tra il recitativo (ossia il lungo preambolo con cui l'attore si presenta alla sala) e l'aria. E il suono si vuole regionale, si pensi al vernacolo toscano, a Benigni, esploso anche grazie al cinema, a quello lombardo di Paolo Rossi sulla scia di Fo, a quello genovese di Beppe Grillo. Ma questi one man show tendono ad abolire conflitti interpersonali, soli come sono, e si presentano in quanto performer, senza più impegnarsi nella costruzione del personaggio. Sono se stessi e basta.

PUPPA, P. The actor and the street. Trans/Form/Ação (São Paulo), v.24, p.47-56, 2001.

- ABSTRACT: This article deals with the following topics: trajectory of the political theater from 1968 to the end of the 80s; actions in schools and factories; inventory of the american tours in Italy; discovery of the third theater; Dario Fo and the displacement of space; end of political activism replaced by media imperialism; the actor-narrator in the deideologizing return.

- KEYWORDS: Political theater; third theater; Dario Fo. 